\title{
Diversidad taxonómica y funcional de aves: Diferencias entre hábitats antrópicos en un bosque subtropical
}

\author{
Anahí S. Vaccaro ${ }^{\bowtie}$ \& M. Isabel Belloce \\ Departamento de Ecología, Genética y Evolución, IEGEBA (CONICET-UBA), Facultad de Ciencias Exactas y Naturales, \\ Universidad de Buenos Aires, Buenos Aires, Argentina.
}

\begin{abstract}
Resumen. Una de las amenazas principales para la biodiversidad es el avance de las actividades humanas que implican usos extensivos de la tierra. En este estudio buscamos identificar los usos de la tierra que mejor preservan el ensamble de especies de aves y sus rasgos funcionales en un bosque subtropical. Para ello comparamos simultáneamente la diversidad taxonómica (riqueza específica y similitud en la composición de especies) y la funcional (diversidad de rasgos funcionales y similitud en la composición de rasgos) de aves entre el bosque nativo y los hábitats antrópicos que resultan de actividades como las forestaciones, la urbanización, la ganadería y la agricultura, en uno de los bosques subtropicales más diversos y amenazados del mundo: el Bosque Atlántico del Alto Paraná. Relevamos aves y variables ambientales en los cinco tipos de hábitat, y seleccionamos 11 rasgos para estimar la diversidad funcional. Estimamos el "1-índice de disimilitud de Gower" para explorar las similitudes entre el bosque nativo y los hábitats antrópicos. Pusimos a prueba modelos de cuadrados mínimos generalizados para comparar la similitud ambiental y las diversidades taxonómica y funcional entre los tipos de hábitat. Estudiamos la asociación de los rasgos funcionales en cada tipo de hábitat mediante un fourth corner analysis. Como esperábamos, las forestaciones fueron el hábitat que mejor preservó el ensamble de aves y de rasgos funcionales del bosque nativo, y los usos de la tierra que resultan en hábitats abiertos fueron los más diferentes al bosque nativo en diversidad taxonómica y funcional. La similitud ambiental entre los hábitats antrópicos y el hábitat nativo es clave para conservar las aves y sus rasgos funcionales. Estudiar la diversidad taxonómica y funcional en estos hábitats es esencial para predecir las respuestas de las comunidades a los usos de la tierra y permite tomar decisiones de manejo adecuadas.
\end{abstract}

[Palabras clave: agricultura, conservación, filtrado ambiental, forestaciones, ganadería, rasgos funcionales, riqueza específica, diversidad beta, urbanización]

\begin{abstract}
Taxonomic and functional bird diversity: Differences between anthropogenic habitats in a subtropical forest. The extensive land use for human activities is one of the main threats to biodiversity. In this study, we investigate what anthropogenic habitat that best preserves the native assemblage of birds and their functional traits in a subtropical forest. We compared simultaneously the taxonomic (species richness and composition similarity) and functional (functional traits diversity and traits composition similarity) bird diversity between native forest and different anthropogenic habitats (tree plantations, urban centers, cattle pastures and crop fields) in one of the most diverse and threatened forest in the world, the Upper Parana Atlantic Forest. We surveyed birds and measured environmental variables in five anthropogenic habitat types, and selected 11 traits to estimate functional diversity. We estimated "1-Gower dissimilarity index" to explore similarities between native forest and anthropogenic habitats. We used generalized least square models to compare environmental similarity and taxonomic and functional diversity between habitat types. We performed fourth corner analysis to study functional traits associations with each anthropogenic habitat. As expected, tree plantation was the habitat that best preserved bird and functional trait assemblages of the native forest; in contrast, open habitats differed the most from the native forest with respect to taxonomic and functional diversity. Environmental similarity between anthropogenic and native habitats is a key to bird conservation and functional traits. Studying taxonomic and functional diversity in these habitats is essential to predict community responses to land use and allows to make suitable conservation decisions.
\end{abstract}

[Keywords: crop fields, conservation, environmental filtering, tree plantations, cattle pastures, functional traits, species richness, beta diversity, urbanization]

\section{INTRODUCCIÓN}

Los cambios en la biodiversidad debido a las actividades humanas sucedieron más rápidamente en los últimos 50 años que en cualquier otro tiempo en la historia. Una de las principales causas es la modificación de los hábitats nativos por sistemas productivos y urbanos (Millennium Ecosystem Assessment 2005), lo que resulta en nuevos ecosistemas con combinaciones de especies y abundancias que no ocurrieron antes dentro del bioma (Morse et al. 2014). En particular, en los bosques tropicales y subtropicales, estas modificaciones 
son trascendentes ya que la degradación y el reemplazo de los ambientes nativos ocurre de manera acelerada y allí se concentra la mayor parte de las especies del mundo (Myers et al. 2000). Debido a estas modificaciones y a la escasa extensión de las áreas protegidas, para contribuir a la conservación de los bosques tropicales y subtropicales resulta fundamental comprender los mecanismos involucrados en la formación de ensambles en los hábitats generados por las actividades humanas. El estudio de la influencia de varios usos de la tierra sobre la diversidad taxonómica de aves y la diversidad funcional en bosques tropicales y subtropicales posee gran relevancia en la actualidad (Sayer et al. 2017; Chapman et al. 2018) y es esencial para predecir las respuestas de las comunidades a los cambios ambientales y tomar decisiones de manejo adecuadas (Hausner et al. 2003; Waltert et al. 2004). Sin embargo, existen pocos estudios que investigan el grado de influencia de los hábitats antrópicos en las comunidades nativas y en la diversidad funcional en relación a su similitud con el bosque nativo (Sekercioglu 2012; Tinoco et al. 2018).

La diversidad funcional fue definida por primera vez como el valor, rango, distribución y abundancia relativa de los rasgos funcionales que relacionan a los organismos con las funciones del ecosistema (Díaz and Cabido 2001; Tilman 2001). Pero luego, Petchey et al. (2004) extendieron el concepto para abarcar los rasgos de historia de vida relacionados con la capacidad de los organismos para vivir en un ambiente determinado y responder a los cambios ambientales tanto naturales como antrópicos. El concepto de "rasgo" varía en la literatura y se lo suele definir como características morfológicas, ecológicas, fisiológicas o fenológicas medibles a nivel individual (Violle et al. 2007); no obstante, también se utilizaron características a nivel de especie (Casanoves et al. 2011; Luck et al. 2012) como la distribución geográfica, estatus migratorio o abundancia regional (Cofre et al. 2007; Newbold et al. 2014). Los rasgos funcionales pueden influir en la tolerancia ambiental y requerimientos de hábitat, y determinan dónde pueden vivir las especies, cómo interactúan con otras, y cuál es su contribución al funcionamiento de los ecosistemas (Cadotte et al. 2011). El estudio de la diversidad funcional permite entender las respuestas de las especies a los cambios ambientales (Schleuter et al. 2010; Cadotte et al. 2011) y el proceso de formación de ensambles (Petchey and Gaston 2007; Cornwell and Ackerly 2009).

Las condiciones ambientales y disturbios antrópicos influyen en la diversidad funcional porque resultan en la pérdida o adición de especies con ciertos rasgos funcionales (Vandewalle et al. 2010; Luck et al. 2013). Esto se aprecia con claridad en las comunidades de aves porque poseen una gran variedad de funcionales ecológicas (Sekercioglu 2006) y son sensibles a los cambios en la estructura de la vegetación (MacArthur and MacArthur 1961) y a las modificaciones humanas del hábitat (Lawton et al. 1998; Sekercioglu et al. 2004). Por otro lado, las aves desempeñan funciones clave en la dinámica de los bosques (e.g., depredación de insectos herbívoros, dispersión de semillas, polinización) y son consideradas "enlaces móviles" que favorecen la resiliencia de los bosques al cambio antrópico (Lundberg and Moberg 2003; Sekercioglu 2006).

La diversidad taxonómica y funcional de los ensambles de aves en un determinado hábitat antrópico dependerá de cómo los usos de la tierra alteren los filtros ambientales naturales impuestos al conjunto regional de especies (Temperton 2004; Birkhofer et al. 2017). Kraft et al. (2015) definen el filtrado ambiental sensu stricto como los recursos y las condiciones ambientales que excluyen especies con límites fisiológicos oecológicosinviables, evitandoque se establezcan o persistan en un determinado lugar. Nuestra hipótesis es que aquellos usos de la tierra que implican cambios drásticos en la estructura de la vegetación provocan una pérdida de diversidad taxonómica y funcional nativa (Flynn et al. 2009; Mayfield et al. 2010) mayor que otros usos que mantienen una estructura similar de la vegetación (Gascon et al. 1999; Zurita and Bellocq 2010). Por lo tanto, esperamos que las diversidades taxonómica y funcional en los hábitats antrópicos dependan de la similitud ambiental entre estos hábitats y el bosque nativo (Filloy et al. 2010; Diniz-Filho et al. 2011).

Nuestros objetivos son 1) identificar los hábitats antrópicos que preservan mejor el ensamble de especies nativas y los rasgos funcionales de aves que ocurren en el Bosque Atlántico del Alto Paraná, uno de los biomas menos estudiados y con mayores pérdidas de hábitat por el avance de la frontera agropecuaria (Myers et al. 2000; Ribeiro et al. 2009). Para ello comparamos la diversidad taxonómica (riqueza específica y similitud en la composición de especies) y la 
diversidad funcional (diversidad, divergencia y equitatividad de rasgos funcionales, y similitud en la composición de rasgos) de aves entre el bosque nativo (mantenido por las áreas protegidas) y hábitats antrópicos (resultantes de forestaciones, urbanización, ganadería y agricultura). En base a nuestra predicción y a estudios previos (Peh et al. 2006; Zurita and Bellocq 2012), esperamos que debido a la presencia de un estrato arbóreo continuo, las forestaciones sean más similares al bosque nativo en su diversidad taxonómica y funcional que los otros hábitats antrópicos. Sin embargo, los centros urbanos pueden tener un porcentaje de cobertura arbórea que permita la presencia de algunas especies nativas y rasgos funcionales (Filloy et al. 2018) y por ello esperamos que presenten mayor similitud al bosque nativo que los sitios ganaderos y agrícolas con vegetación monoestratificada. Por otra parte, 2) estudiamos qué rasgos funcionales se asocian a cada tipo de hábitat y profundizamos en las causas de diferenciación entre los ensambles para conocer los rasgos que pueden permitir la tolerancia de las especies a las características ambientales y para indagar acerca de las consecuencias de la pérdida o la adición de especies con ciertos rasgos funcionales en el funcionamiento de los ecosistemas.

\section{MAteriales y Métodos}

\section{Diseño de estudio}

Para identificar los hábitats antrópicos que preservan mejor la diversidad del bosque nativo, relevamos aves y variables ambientales en hábitats naturales de bosque y en cuatro tipos de hábitat antrópico en el Bosque Atlántico del este de Paraguay. Seleccionamos en total 22 sitios (Figura 1), que consistieron en tres bosques nativos, cinco forestaciones, cuatro centros urbanos, cinco sitios ganaderos y cinco sitios agrícolas. El número de sitios por cada tipo de hábitat fue desbalanceado porque priorizamos la elección de sitios representativos de los diferentes usos de la tierra y a los cuales fuera posible acceder. Cada sitio consistió en un área con tamaño mínimo de 20 ha y un mismo uso de la tierra. Los sitios estaban separados al menos $2 \mathrm{~km}$ para asegurar comunidades locales diferentes. En cada sitio registramos la cobertura y estratificación de la vegetación para estimar la similitud ambiental con el bosque nativo. Estimamos la riqueza

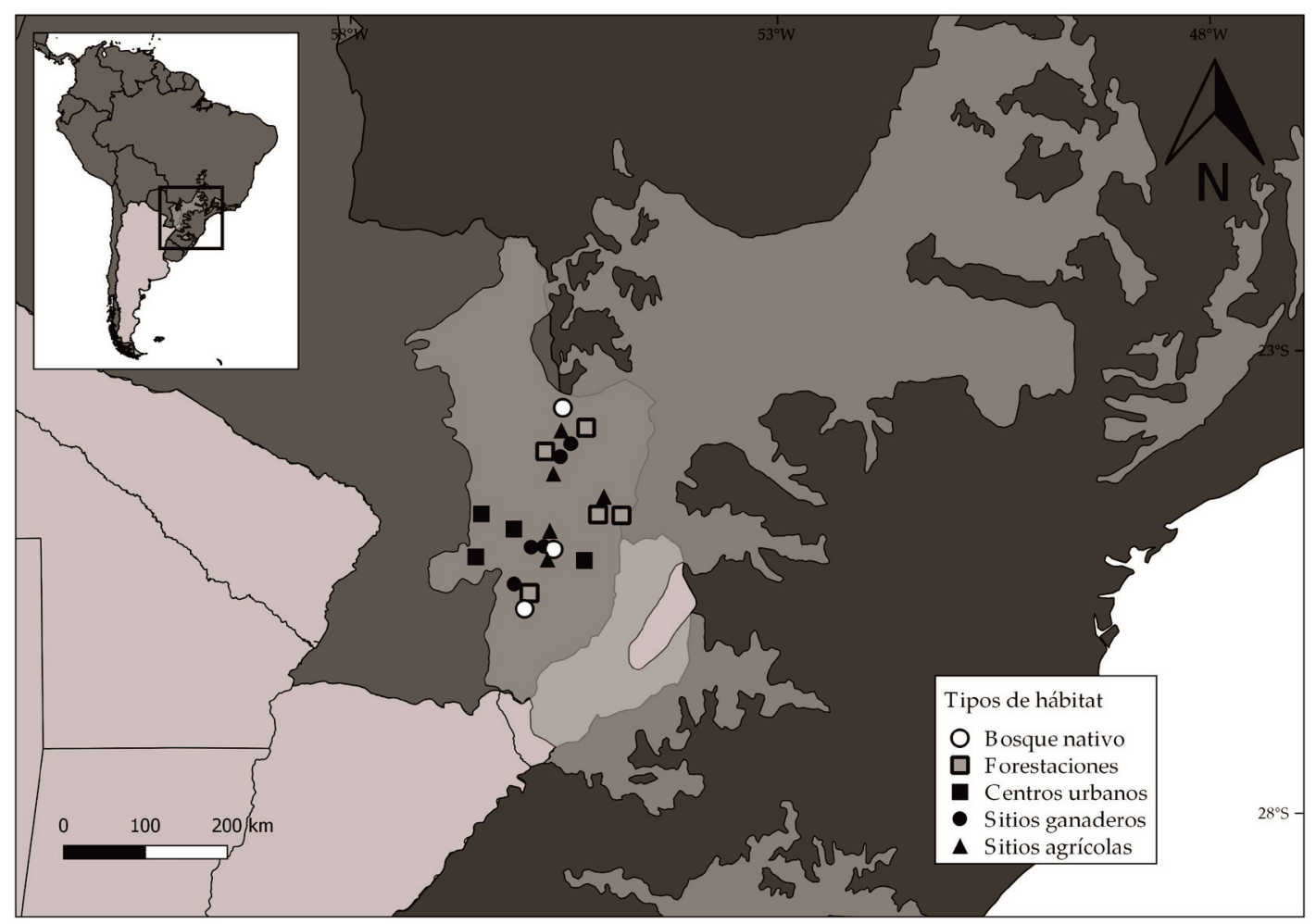

Figura 1. Ubicación de los sitios de muestreo en distintos tipos de hábitat en el Bosque Atlántico en Paraguay.

Figure 1. Location of sampling sites in different habitat types in the Atlantic Forest in Paraguay. 
y similitud taxonómica, y seleccionamos 11 rasgos funcionales (ver Tabla B en el anexo) para estimar índices de diversidad y similitud funcional para cada sitio de estudio.

\section{Área de estudio}

El Bosque Atlántico es uno de los cinco principales focos de biodiversidad del mundo y conserva sólo el 7\% de su cobertura forestal original (Myers et al. 2000). El clima de la región es subtropical y la vegetación predominante es bosque semi-deciduo formado por tres estratos arbóreos con numerosas epífitas y lianas, cañas y helechos arborescentes (Di Bitetti et al. 2003). En los remanentes del bosque nativo del este de Paraguay, el dosel está dominado por árboles de las familias Myrtaceae, Lauraceae y Fabaceae, mientras que el sotobosque está cubierto principalmente por helechos y bambúes (Oliveira-Filho and Fontes 2000). De la cobertura de bosque nativo original, sólo 17\% permanece como áreas protegidas o fragmentos en tierras privadas (Galindo Leal and de Gusmão Câmara 2003). El 90\% de la superficie total de Paraguay está dedicada a la agricultura y la ganadería (Di Bitetti et al. 2003; Zurita and Bellocq 2010). En este sector se concentra $\sim 80 \%$ de la superficie sembrada con soja del país (INBIO 2008). Además, Paraguay cuenta con $\sim 66000$ ha de plantaciones forestales, de las cuales $50 \%$ se implantó a partir de la Ley de Fomento a la Forestación y Reforestación (Instituto Forestal Nacional 2013).

Seleccionamos sitios dedicados a la agricultura (con cultivos de soja), sitios ganaderos compuestos por pastos y herbáceas dicotiledóneas, y forestacionesconplantaciones comerciales de eucalipto (principalmente Eucalyptus grandis) de 7 a 10 años de edad destinado a producir madera y pulpa. Los centros urbanos elegidos pertenecían a ciudades de tamaño intermedio (entre 14000 y 60000 habitantes), con más de $70 \%$ de la superficie con cobertura impermeable (i.e., edificios, casas, calles pavimentadas). Las tres áreas protegidas que representaron el bosque nativo fueron: Reserva Natural Mbaracayú (64405 ha), Reserva Natural Ypetí (13592 ha) y Reserva Natural Tapytá (4737 ha) (Tabla A, Anexo).

\section{Muestreo}

En cada sitio de estudio establecimos 10 estaciones de muestreo de aves y variables ambientales, alejadas al menos $200 \mathrm{~m}$ de otros tipos de hábitat. La distancia mínima entre estaciones de muestreo fue $150 \mathrm{~m}$ para minimizar posibles sesgos por doble conteo de aves (Bibby et al. 1998). Realizamos los muestreos durante la primavera (septiembre a noviembre) de 2015. Para el muestreo de aves utilizamos el método de conteo por puntos con radio fijo de $50 \mathrm{~m}$ (Ralph et al. 1996). En cada punto de conteo, dos observadores registraron de forma simultánea e independiente todas las aves vistas y oídas durante 5 minutos, desde el amanecer y por aproximadamente 4 horas, en días soleados y de viento leve o moderado. Las aves de paso que volaban por encima del área no fueron consideradas (Ralph et al. 1996). Grabamos los cantos de aves utilizando un grabador digital (Zoom H4next Handy Recorder) con el volumen de grabación calibrado por sitio para registrar sonidos hasta $50 \mathrm{~m}$. El proceso de identificación de aves culminó luego del trabajo de campo y con el análisis de un total de 590 grabaciones, utilizando grabaciones publicadas para la verificación de las especies (Xeno-canto-Foundation 2018).

Medimos las siguientes variables ambientales relacionadas a la cobertura y estructura de la vegetación en cada estación de muestreo: porcentaje de cobertura de árboles, arbustos, vegetación herbácea (incluyendo pastos, dicotiledóneas y cultivos) y altura de los pastos. Además, registramos variables relacionadas con el suelo como cobertura de suelo desnudo, broza, y área impermeable. Consideramos a los cultivos como parte del estrato herbáceo porque nuestro objetivo fue realizar una caracterización general de la estructura de la vegetación. En cada estación de muestreo establecimos al azar tres cuadrantes de $1 \mathrm{~m}^{2}$ dentro del área de $50 \mathrm{~m}$ de radio donde registramos de forma visual la cobertura de arbustos, pastos, suelo desnudo y broza siguiendo el procedimiento de Braun Blanquet (Mueller-Dombois and Ellenberg 1974). Los valores de los tres cuadrantes se promediaron para obtener un valor por estación de muestreo. Para estimar la cobertura de árboles y de área impermeable, utilizamos imágenes satelitales del círculo con radio de $50 \mathrm{~m}$ para cada estación de muestreo. Para cada una de las variables ambientales, promediamos los valores de las 10 estaciones de muestreo por sitio.

\section{Selección de rasgos funcionales}

Seleccionamos 11 rasgos funcionales (y sus categorías) relacionados con la historia de vida 
de las especies basados en estudios previos de diversidad funcional y de las respuestas al reemplazo del hábitat natural (e.g., Cofre et al. 2007; Flynn et al. 2009; Vandewalle et al. 2010; Corbelli et al. 2015) (Tabla B, Anexo). Estos rasgos son relevantes para entender cómo las especies de aves responden a los cambios ambientales y cuál es su contribución al funcionamiento de los ecosistemas (Luck et al. 2012, 2013). Todos fueron categóricos y cada categoría fue binaria: asignamos 1 ó 0 dependiendo de si la especie presenta o no esa categoría de rasgo. Por ejemplo, el rasgo dieta fue subdividido en tantas categorías como estados; para una especie que consume principalmente insectos y semillas, se le asignó 1 a esas categorías y 0 al resto de las categorías. Todas las categorías de un mismo rasgo fueron mutuamente excluyentes (sólo una categoría podía tener valor 1), excepto para dieta, estrato de forrajeo y hábitat principal de anidamiento. La información para asignar las categorías de los rasgos fue extraída de Narosky et al. (2010), de la Peña $(2013,2016)$, del Hoyo et al. (2016), BirdLife International (2018) y López-Lanús et al. (2008).

\section{Análisis de datos}

Con los datos relevados y los valores de rasgos funcionales asignados, construimos tres matrices de datos: (1) sitios por variables ambientales, (2) sitios por especies, y (3) especies por rasgos. Para la matriz (1), para cada una de las variables ambientales calculamos el valor promedio por sitio (utilizando los datos obtenidos en las 10 estaciones de muestreo), obteniendo una matriz de dimensiones 22x7. La matriz de sitios por especies fue construida sumando las abundancias de las especies registradas en los 10 puntos de conteo para cada sitio de estudio, obteniendo una matriz con dimensiones 22x150 (porque en total se registraron 150 especies de aves). Por último, la matriz de especies y rasgos tuvo 150x55 dimensiones porque seleccionamos en total 55 categorías de rasgos funcionales para cada especie (Tabla B, Anexo).

Para cada una de las variables ambientales, calculamos el valor promedio de los sitios de bosque nativo, y para cada tipo de hábitat antrópico, calculamos la similitud ambiental entre cada sitio y el promedio de los bosques nativos utilizando "1-índice de distancia de Gower" con la función vegdist en el paquete "vegan" (Oksanen et al. 2016). Calculamos la riqueza de especies de aves en cada sitio de estudio (Magurran 2004) con la función "diversityresult" en el paquete "BiodiversityR" (Kindt and Coe 2005). Para cada sitio, estimamos tres métricas de diversidad funcional que tienen en cuenta la abundancia de las especies (Mason et al. 2005; Luck et al. 2013): la equitatividad funcional (FEve) analiza la regularidad en la que se distribuyen las especies en el espacio funcional, la divergencia funcional (FDiv) cuantifica cuán lejos se encuentran las especies más abundantes del centro del espacio funcional (Villéger et al. 2008), y la dispersión funcional (FDis) que es la distancia media de las especies individuales al centroide del espacio funcional (Laliberté and Legendre 2010). Todas las métricas fueron calculadas con el paquete "FD" (Laliberté and Legendre 2010).

Para obtener la similitud taxonómica y funcional entre el ensamble de aves del bosque nativo y los ensambles de cada tipo de hábitat antrópico, a partir de la matriz (2) de sitios por especies construimos una matriz de sitios y presencia/ausencia de especies, con las especies registradas en los bosques nativos agrupadas en una sola columna para mejorar la representación del ensamble de aves nativas y sus rasgos (Garaffa et al. 2009). Para calcular la similitud en la composición de especies estimamos "1 - índice de disimilitud de Jaccard" entre los sitios de estudio usando el paquete "betapart" (Baselga and Orme 2012). El índice de Jaccard originalmente fue utilizado como índice de similitud (Jaccard 1912) pero en el paquete "vegan" (utilizado en el "betapart") fue adaptado para ser expresado como índice de disimilitud. Para estimar la similitud funcional entre los ensambles de aves de cada tipo de hábitat antrópico y el ensamble de aves del bosque nativo, seguimos el procedimiento descripto por Swenson et al. (2011) y calculamos un dendrograma que presenta la similitud entre especies respecto a los rasgos. Con el dendrograma y con la matriz de sitios y presencia/ausencia de especies calculamos el índice funcional de Sorensen (FSor). FSor es un indicador de las funciones compartidas entre dos comunidades (Swenson 2011), y es análogo a la métrica filogenética PhyloSor del paquete "PICANTE" (Bryant et al. 2008).

Empleamos modelos de cuadrados mínimos generalizados (GLS) para poner a prueba las diferencias entre los tipos de hábitat con respecto a la similitud ambiental, riqueza 
de especies, diversidad funcional, similitud taxonómica y similitud funcional. Utilizamos la función "gls" del paquete "nlme" (Pinheiro et al. 2014) y modelamos varianzas con la función "varIdent" en los casos que fuera necesario. Las comparaciones a posteriori se hicieron con la prueba de Tukey con la función "glht" en el paquete "multcomp" (Hothorn et al. 2008). Por último, utilizando las matrices de sitios $\mathrm{x}$ tipo de hábitat, sitios $\mathrm{x}$ especies $\mathrm{y}$ especies $x$ rasgos realizamos un fourth-corner analysis (Brown et al. 2014) para analizar la asociación de los rasgos funcionales con cada tipo de hábitat. Para mejorar la visualización de los gráficos, se realizaron cuatro análisis por separado con los grupos de rasgos detallados en la Tabla B del anexo. Todos los análisis estadísticos se llevaron a cabo utilizando el software R (R Core Team 2018) y consideramos diferencias significativas con un valor de $P<0.05$.

\section{Resultados}

En los 22 sitios muestreados registramos un total de 2162 individuos correspondientes a 150 especies: 276 individuos de 64 especies en el bosque nativo, 463 individuos de 58 especies en las forestaciones, 628 individuos de 31 especies en los centros urbanos, 549 individuos de 69 especies en los sitios ganaderos y 246 individuos de 30 especies en los sitios agrícolas (Tabla C, Anexo).

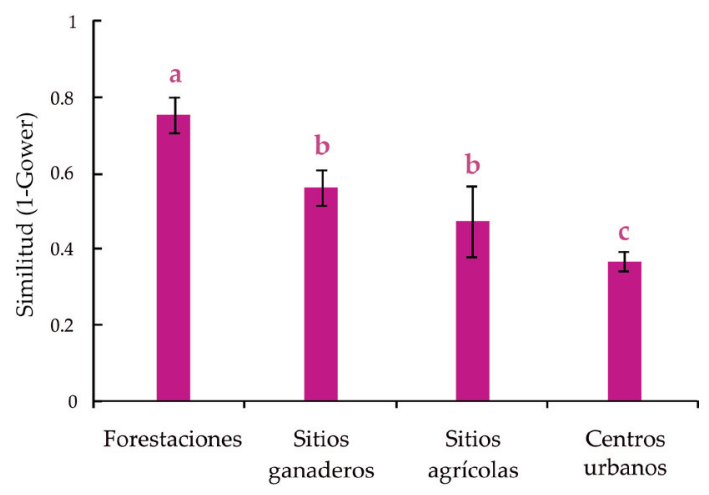

Figura 2. Valores promedio y desvío estándar de la similitud ambiental (1-índice de Gower) calculada entre el bosque nativo y cada tipo de hábitat antrópico. Letras diferentes representan diferencias significativas entre tipos de hábitat determinadas por la prueba de Tukey de comparaciones a posteriori $(P<0.05)$.

Figure 2. Averages and standard deviations of environmental similarity (1-Gower index) calculated between native forest and each anthropogenic habitat type. Different letters indicate significant differences between habitat types resulting of posteriori comparisons Tukey test $(P<0.05)$.
La similitud ambiental con el bosque nativo difirió entre los tipos de hábitats antrópicos $\left(\mathrm{F}_{3,15}=33.47, P<0.0001\right)$. Las forestaciones tuvieron significativamente la mayor similitud, seguidas por los sitios ganaderos y agrícolas con similitud intermedia y sin diferencias significativas entre sí (Figura 2). Por último, los centros urbanos mostraron significativamente la menor similitud.

La riqueza de especies difirió significativamente entre los tipos de hábitat $\left(\mathrm{F}_{4,17}=7.72, P=0.001\right)$, presentando mayores valores en el bosque nativo, sitios ganaderos $y$ las forestaciones (Figura 3). Luego siguieron los centros urbanos, significativamente diferentes del bosque nativo y sitios ganaderos, pero no de las forestaciones ni de los sitios agrícolas.

Los tipos de hábitat difirieron significativamente en FDis $y$ FEve $\left(\mathrm{F}_{4,17}=206.25, P<0.0001 ; \mathrm{F}_{4,17}=8.753, P<0.001\right.$; respectivamente), peronoen $\mathrm{FDiv}\left(\mathrm{F}_{4,17}=0.4878\right.$, $P=0.75)$. El orden decreciente de los valores promedio de FDis fue: forestaciones, sitios ganaderos, bosque nativo, sitios agrícolas y centros urbanos; y para los valores de FEve: forestaciones, bosque nativo, sitios agrícolas, sitios ganaderos y centros urbanos (Figura 4). Las forestaciones tuvieron los mayores valores de FDis, significativamente diferentes a los demás tipos de hábitat (Figura 4). Los sitios ganaderos, bosque nativo y sitios agrícolas no difirieron significativamente entre sí. Para FEve hubo diferencias significativas entre los centros urbanos y el bosque nativo y las forestaciones (Figura 4).

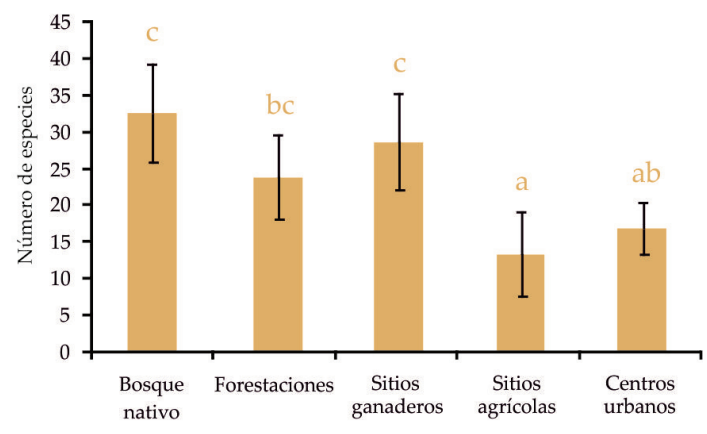

Figura 3. Valores promedio y desvío estándar de la riqueza de especies en cada tipo de hábitat. Letras diferentes indican diferencias significativas entre los tipos de hábitat determinadas por la prueba de Tukey de comparaciones a posteriori $(P<0.05)$.

Figure 3. Averages and standard deviations of species richness in each habitat type. Different letters indicate significant differences between habitat types resulting of posteriori comparisons Tukey test $(P<0.05)$. 


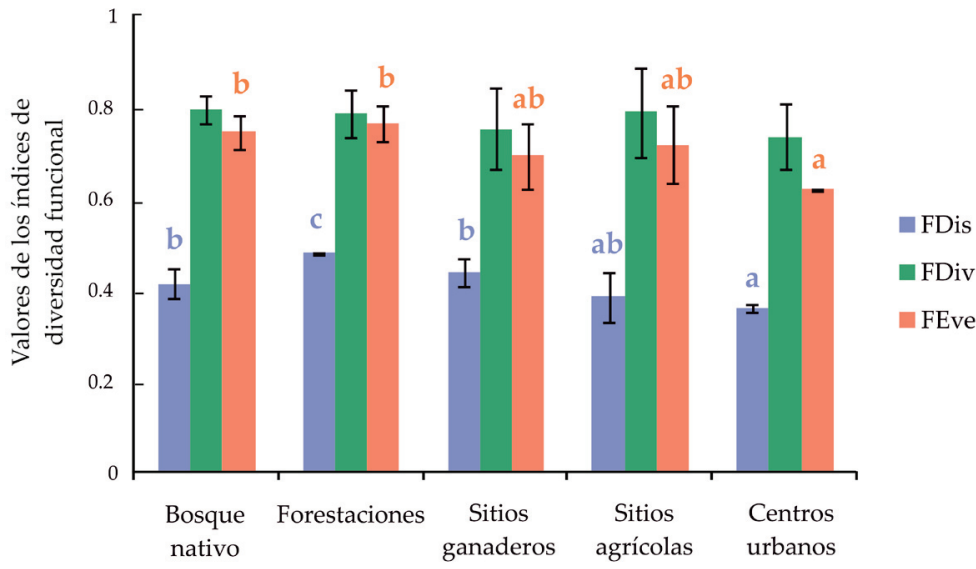

Figura 4. Valores promedio y desvío estándar de los índices FDis, FDiv y FEve para los sitios de cada tipo de hábitat.Letrasdiferentesrepresentan diferencias significativas entre tipos de hábitat determinadas por la prueba de Tukey de comparaciones a posteriori $(P<0.05)$.

Figure 4. Averages and standard deviations of FDis, FDiv and FEve index to each habitat type sites. Different letters indicate significant differences between habitat types resulting of posteriori comparisons Tukey test $(P<0.05)$.
Los tipos de hábitat antrópicos difirieron entre sí en su similitud con el bosque nativo tanto en composición de especies $\left(\mathrm{F}_{3,15}=24.63\right.$, $P<0.0001)$ y de rasgos funcionales $\left(\mathrm{F}_{3,15}=50.628\right.$, $P<0.0001)$. Los órdenes decrecientes de similitud promedio fueron: forestaciones, centros urbanos, sitios ganaderos y sitios agrícolas para la composición taxonómica; forestaciones, sitios ganaderos, centros urbanos y sitios agrícolas para la composición funcional (Figura 5). En ambos casos, las forestaciones fueron el tipo de hábitat con significativamente la mayor similitud con el bosque nativo (Figura 5). La similitud entre los sitios agrícolas y el bosque nativo fue la más baja, aunque sin diferencias significativas con la similitud entre los sitios ganaderos y el

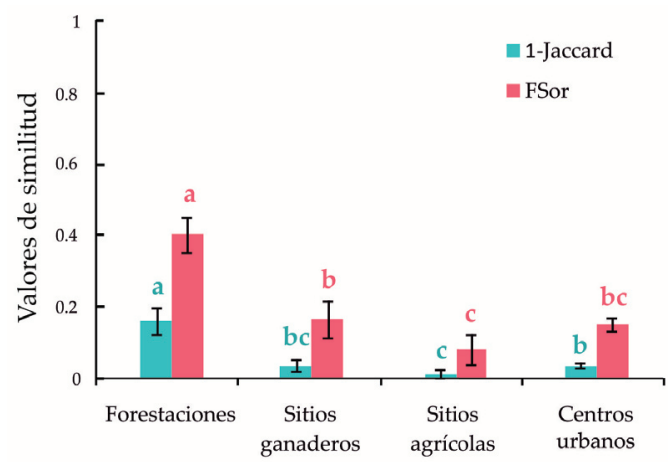

Figura 5. Valores promedio y desvío estándar de las similitudes taxonómica y funcional (1-índice de Jaccard e índice FSor, respectivamente) calculadas entre el bosque nativo y cada tipo de hábitat antrópico. Letras diferentes representan diferencias significativas entre tipos de hábitat determinadas por la prueba de comparaciones a posteriori de Tukey $(P<0.05)$.

Figure 5. Averages and standard deviations of taxonomic and functional similarity (1-Jaccard index and FSor index, respectively) calculated between native forest and each anthropogenic habitat type. Different letters indicate significant differences between habitat types resulting of posteriori comparisons Tukey test $(P<0.05)$. bosque nativo en la composición taxonómica ni con los valores de similitud entre centros urbanos y bosque nativo en la composición funcional (Figura 5).

Cada hábitat resultó asociado a diferentes rasgos funcionales (Figura 6). El bosque nativo estuvo asociado a rasgos relacionados al uso de estratos de forrajeo arbóreo y arbustivo, y a aves especialistas de bosque, sensibles al disturbio antrópico y que son insectívoras, frugívoras o granívoras. Los sitios forestales tuvieron una asociación positiva con rasgos asociados al estrato arbóreo, al forrajeo generalista y abundancia frecuente, y asociaciones leves con el uso de estrato arbustivo. Los sitios agrícolas y ganaderos mostraron asociación positiva con rasgos relacionados al uso del suelo y del estrato herbáceo (forrajeo y nidificación) $y$ asociaciones negativas con rasgos de forrajeo en árboles, arbustos, dieta frugívora y nectarívora. Los centros urbanos tuvieron una asociación positiva con la distribución cosmopolita, con rasgos generalistas, con rasgos especialistas del hábitat urbano (e.g., nidificación en edificios) y favorecidas por el disturbio antrópico.

\section{DISCUSIÓN}

Las forestaciones fueron el tipo de hábitat que mostró las mayores similitudes con el ensamble de especies y rasgos funcionales encontrados en el bosque nativo; por el contrario, los usos de la tierra que resultan en hábitats abiertos sin estrato arbóreo fueron los más contrastantes. Nuestros resultados son consistentes con estudios previos que documentaron que los usos agroforestales, en comparación con la agricultura y ganadería, generan hábitats antrópicos más adecuados 


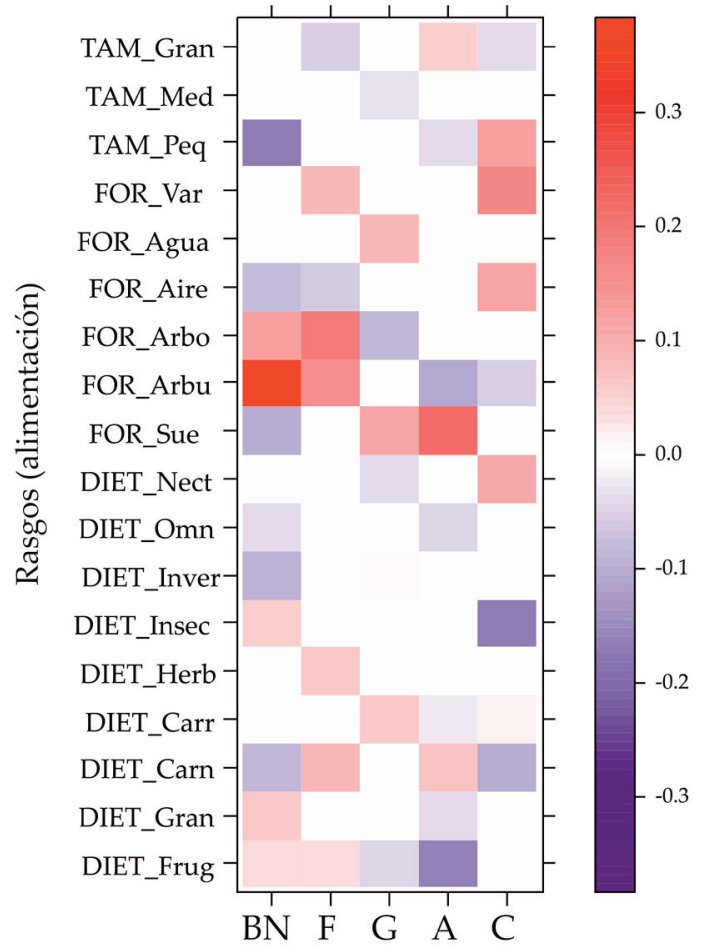

Tipos de hábitat

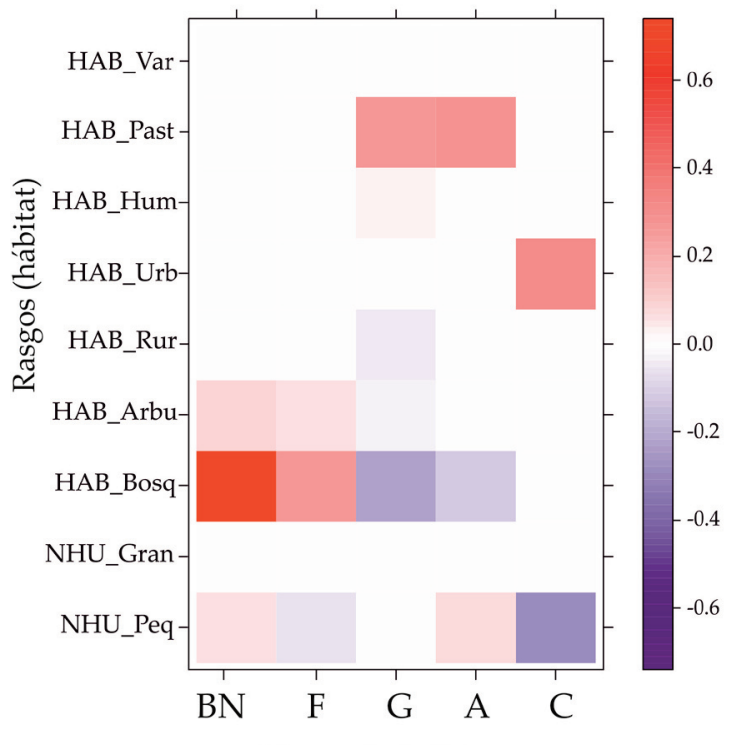

Tipos de hábitat

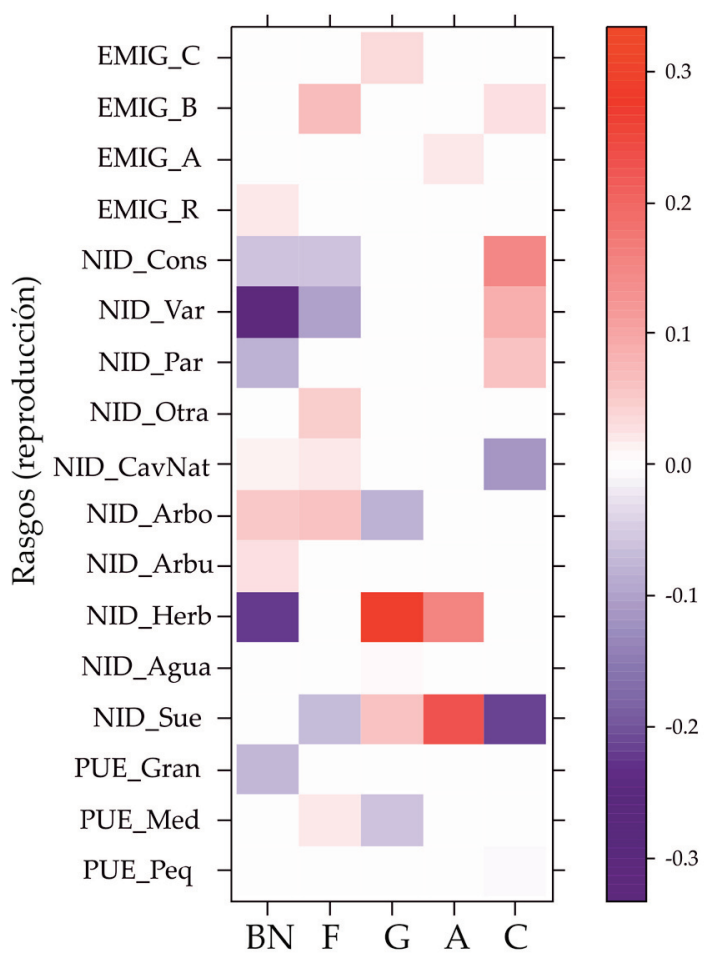

Tipos de hábitat

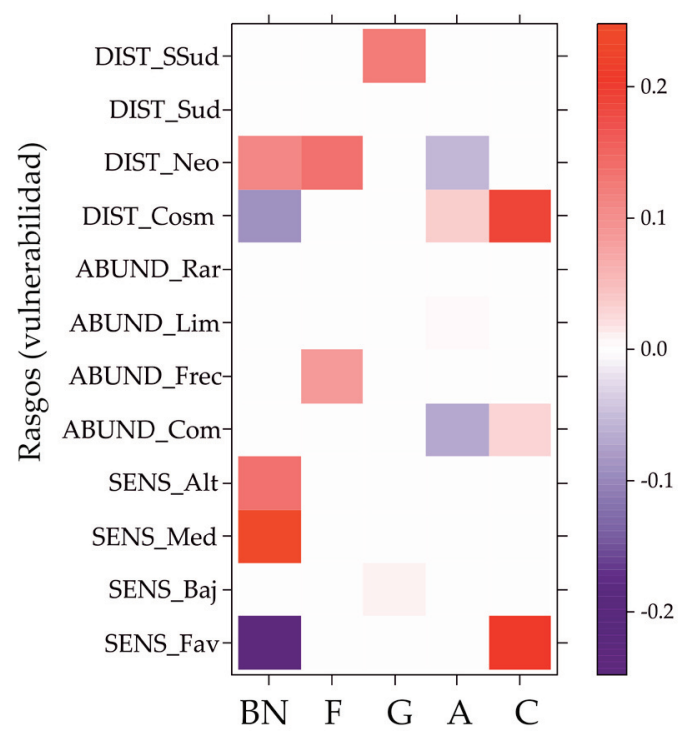

Tipos de hábitat

Figura 6. Resultados del fourth corner analysis para los grupos de rasgos funcionales y cada tipo de hábitat. Los tipos de hábitat son: bosque nativo $(\mathrm{BN})$, forestaciones $(\mathrm{F})$, sitios ganaderos $(\mathrm{G})$, sitios agrícolas $(\mathrm{A})$ y centros urbanos $(\mathrm{C})$. Los acrónimos y grupos de los rasgos se encuentran en la Tabla B del anexo. Los cuadrados más brillantes muestran asociaciones fuertes y los cuadrados más pálidos asociaciones más débiles. Las asociaciones positivas son rojas y las negativas son azules.

Figure 6. Fourth-corner analysis results for functional traits groups and each habitat type. Habitat types are: native forest $(B N)$, tree plantations $(F)$, cattle pastures $(G)$, crop fields (A), urban centers $(C)$. Acronyms of traits and functional traits groups are given in Table $\mathrm{B}$ in the appendix. Brighter squares show strong associations and paler squares show weak associations. Positive associations are red and negative associations are blue 
para las especies nativas en el Bosque Atlántico y otros bosques tropicales (Peh et al. 2006; Zurita and Bellocq 2012). Además, según nuestro estudio, el uso forestal es el que menor impacta negativamente en la diversidad funcional del bosque nativo.

De acuerdo con nuestra predicción, las forestaciones fueron el tipo de hábitat que mejor preservó el ensamble de especies y rasgos funcionales del bosque nativo. Esto es porque las forestaciones (incluso de especies exóticas) en un bioma de bosque resultan en la imposición de filtros ambientales menos intensos que en otros tipos de hábitat como campos agrícolas o ganaderos. Por ejemplo, permiten el ingreso de especies de aves que se alimentan en el dosel (Loyn et al. 2007), aunque especies especialistas del bosque nativo y altamente sensibles al disturbio antrópico no suelen utilizar las forestaciones (Petit and Petit 2003). Por otra parte, las plantaciones forestales suelen tener manejos (poda y raleo) que simplifican la estructura de la vegetación, lo que puede explicar por qué no observamos asociación positiva de aves que forrajean en arbustos en las forestaciones, pero si en el bosque nativo.

El bosque nativo presentó los valores mayores de riqueza de especies, pero no de diversidad funcional. Es posible que el espacio multidimensional de rasgos funcionales de las aves en los hábitats naturales sea más pequeño porque en el ambiente nativo predominan las especializaciones (Farwig et al. 2008; Rakotoarisoa and Capparella 2013). Además, los bosques nativos presentaron menor diversidad funcional que las forestaciones. Esto se debe a que en las forestaciones encontramos especies con rasgos similares a las del bosque sumado a especies con rasgos generalistas como las que pueden forrajear y nidificar en varios ambientes.

Los centros urbanos fueron uno de los tipos de hábitat más contrastantes al bosque nativo respecto a la diversidad taxonómica y funcional. Si bien en general las urbanizaciones son ambientes altamente contrastantes al bosque nativo y generan pérdidas en la diversidad, tanto por la marcada reducción de la cobertura de la vegetación (Zipperer et al. 1997) como por la alta cobertura de edificios y pavimento (Palacio et al. 2018), algunas ciudades proveen recursos para especies nativas de bosque al retener especies de árboles del hábitat natural (Filloy et al. 2018). De todos modos, las zonas más arboladas de las ciudades son las residenciales (Zipperer et al. 1997) y no fueron incluidas en este estudio. Los centros urbanos mostraron asociaciones positivas con rasgos funcionales generalistas, cosmopolitas y especialistas de hábitats urbanos y asociaciones negativas con rasgos funcionales relacionados al bosque nativo. El filtrado ambiental que provoca la urbanización permite el ingreso de especies con rasgos para tolerar las condiciones ambientales urbanas (Croci et al. 2008) y evita el establecimiento de muchas especies de aves nativas con rasgos específicos como dieta especialista (e.g. exclusivamente frugívoras) o con alta sensibilidad al disturbio humano (e.g. aves que nidifican en el suelo) (Seress and Liker 2015).

Inesperadamente, los sitios ganaderos presentaron valores altos de riqueza y diversidad funcional con respecto a los demás tipos de hábitat antrópicos. Sin embargo, los sitios ganaderos eran heterogéneos: observamos la presencia de unos pocos árboles o arbustos, zonas bajas inundables, áreas de pastos altos y otras con pastos cortos, lo cual probablemente contribuyó a la presencia de un número de especies y rasgos funcionales relativamente elevado en comparación con otros tipos de hábitat más homogéneos. Estos resultados son consistentes con numerosos estudios que han presentado evidencia que la riqueza aumenta con la heterogeneidad ambiental (Stein et al. 2014). De acuerdo a lo esperado, los sitios agrícolas presentaron riqueza y similitud taxonómica bajas respecto al bosque nativo. Los sitios agrícolas (constituidos principalmente por monocultivos de soja) poseen características ambientales muy diferentes a un bosque, donde la mayoría de las especies del conjunto regional no pueden establecerse (Zurita and Bellocq 2012). Si bien los sitios agrícolas y ganaderos mostraron diversidad funcional intermedia, tal como esperábamos sus similitudes funcionales con el bosque nativo fueron bastante bajas. Los cambios en el uso de la tierra que implican la ausencia de árboles afectan a la diversidad de rasgos funcionales de las aves del bosque nativo (e.g. Sekercioglu et al. 2007; Tscharntke et al. 2008). Tanto la agricultura como la ganadería desarrolladas en el bosque subtropical generan hábitats que imponen filtros ambientales que impiden la persistencia de aves con rasgos funcionales asociados al bosque. Por ejemplo, en nuestros resultados observamos asociaciones negativas con rasgos de forrajeo y nidificación en árboles 
y arbustos. Por otro lado, estos hábitats permiten el ingreso de especies y rasgos nuevos (encontramos asociación positiva con rasgos de nidificación en el suelo y en el estrato herbáceo) y por eso mostraron valores intermedios de diversidad funcional respecto a los otros hábitats.

Las asociaciones negativas de los hábitats antrópicos con algunos rasgos funcionales reflejan una posible pérdida de importantes funciones ecosistémicas para el bosque nativo. Particularmente en las aves, la dieta es uno de los rasgos funcionales de efecto más importante (Newbold et al. 2014). Por ejemplo, las aves frugívoras contribuyen a la dispersión de semillas y las insectívoras al control de plagas (Sekercioglu et al. 2004). Los hábitats antrópicos más contrastantes el bosque nativo mostraron asociaciones negativas con la dieta frugívora, granívora e insectívora. La pérdida de frugívoros en biomas de bosque tropical reduce la distancia de dispersión y reclutamiento de las especies de árboles de grandes semillas, fundamentales para mantener la estructura de la vegetación característica del bosque tropical (Sekercioglu 2006; Wotton and Kelly 2011). Por otro lado, las aves insectívoras que se alimentan de insectos fitófagos promueven el crecimiento de las plantas (Van Bael et al. 2003). En el Bosque Atlántico de Paraguay los hábitats antrópicos contrastantes son los que dominan el paisaje (Di Bitetti et al. 2003; Zurita and Bellocq 2010), y por lo tanto las aves que cumplen estas funciones ecosistémicas quedarían aisladas en los fragmentos de bosque (Bregman et al. 2014).

La similitud ambiental entre los hábitats antrópicos y nativos es un factor clave para contribuir a la conservación de la diversidad taxonómica y funcional de aves del ambiente natural, tanto en bosques tropicales (Sekercioglu 2012; Zurita and Bellocq 2012) como en otros biomas como los pastizales (Azpiroz et al. 2012; Vaccaro et al. 2019). Por ejemplo, así como en el bosque tropical las forestaciones impactan negativamente en menor medida a la avifauna nativa, en pastizales la ganadería puede conservar especies del ensamble de aves nativas (Askins et al. 2007) y sus rasgos funcionales (Vaccaro et al. 2019). Probablemente, el retorno de estos posibles "nuevos ecosistemas" a su estado anterior sea casi imposible (Morse et al. 2014), por lo que es necesario desarrollar manejos en los hábitats antrópicos que permitan mantener características en común con el hábitat nativo y así contribuir a la conservación de la comunidad de aves nativas y sus rasgos funcionales. En el Bosque Atlántico, esto podría contemplar el mantenimiento de remanentes de bosque nativo dentro de los establecimientos agropecuarios (e.g. ley 352/ 94 Paraguay) y corredores de bosque nativo entre hábitats antrópicos (Tabarelli et al. 2010). Además, es fundamental la conservación de los bosques nativos en los cuales habitan especies con rasgos funcionales que no les permiten utilizar hábitats antrópicos.

Agradecimientos. Agradecemos la colaboración de D. Friedrich, A. de Miguel y J. Vrdoljak en los trabajos de campo y a J. Klavins por su trabajo en la identificación de especies y relevamientos. Los muestreos se realizaron gracias a los permisos y apoyo logístico de Pomera Maderas S.A., Campos Morombí, PAYCO S.A., Fundación Moisés Bertoni y GUYRA Paraguay. El editor asociado Pedro G. Blendinger y dos revisores han realizado sugerencias valiosas que mejoraron el manuscrito. También agradecemos el financiamiento provisto por la Agencia Nacional de Promoción Científica y Tecnológica, la Universidad de Buenos Aires y el Consejo Nacional de Investigaciones Científicas y Técnicas.

\section{REFERENCIAS}

Askins, R. A., F. Chávez-Ramírez, B. C. Dale, C. A. Haas, J. R. Herkert, F. L. Knopf, and P. D. Vickery. 2007. Conservation of grassland birds in North America: Understanding ecological processes in different Regions. Ornithological Monographs 64:1-46. https://doi.org/10.2307/40166905.

Azpiroz, A. B., J. P. Isacch, R. A. Dias, A. S. Di Giacomo, C. S. Fontana, and C. M. Palarea. 2012. Ecology and conservation of grassland birds in southeastern South America: a review. Journal of Field Ornithology 83:217-246. https://doi.org/ 10.1111/j.1557-9263.2012.00372.x.

Baselga, A., and C. D. L. Orme. 2012. Betapart: An R package for the study of beta diversity. Methods in Ecology and Evolution 3:808-812. https://doi.org/10.1111/j.2041-210X.2012.00224.x.

Bibby, C., M. Jones, and S. Marsden. 1998. Expedition field techniques: bird surveys. Expedition Advisory Centre, Royal Geographical Society, London, UK.

BirdLife International. 2019. BirdLife International Data Zone. URL: datazone.birdlife.org.

Birkhofer, K., M. M. Gossner, T. Diekötter, C. Drees, O. Ferlian, M. Maraun, S. Scheu, W. W. Weisser, V. Wolters, S. 
Wurst, A. S. Zaitsev, and H. G. Smith. 2017. Land-use type and intensity differentially filter traits in above- and belowground arthropod communities. Journal of Animal Ecology 86:511-520. https://doi.org/10.1111/1365-2656.12641.

Bregman, T. P., C. H. Sekercioglu, and J. A. Tobias. 2014. Global patterns and predictors of bird species responses to forest fragmentation: Implications for ecosystem function and conservation. Biological Conservation 169:372-383. https://doi.org/10.1016/j.biocon.2013.11.024.

Brown, A. M., D. I. Warton, N. R. Andrew, M. Binns, G. Cassis, and H. Gibb. 2014. The fourth-corner solution - using predictive models to understand how species traits interact with the environment. Methods in Ecology and Evolution 5:344-352. https://doi.org/10.1111/2041-210X.12163.

Bryant, J. A., C. Lamanna, H. Morlon, A. J. Kerkhoff, B. J. Enquist, and J. L. Green. 2008. Microbes on mountainsides: contrasting elevational patterns of bacterial and plant diversity. Proceedings of the National Academy of Sciences 105:11505-11511. https://doi.org/10.1073/pnas.0801920105.

Cadotte, M. W., K. Carscadden, and N. Mirotchnick. 2011. Beyond species: Functional diversity and the maintenance of ecological processes and services. Journal of Applied Ecology 48:1079-1087. https://doi.org/10.1111/j.13652664.2011.02048.x.

Casanoves, F., L. Pla, and J. A. Di Rienzo. 2011. Valoración y análisis de la diversidad funcional y su relación con los servicios ecosistémicos. Informe técnico 384. Centro Agronómico Tropical de Investigación y Enseñanza, CATIE Turrialba, Costa Rica.

Chapman, P. M., J. A. Tobias, D. P. Edwards, and R. G. Davies. 2018. Contrasting impacts of land-use change on phylogenetic and functional diversity of tropical forest birds. Journal of Applied Ecology 55:1604-1614. https:// doi.org/10.1111/1365-2664.13073.

Cofre, H. L., K. Böhning-Gaese, and P. A. Marquet. 2007. Rarity in Chilean forest birds: Which ecological and life-history traits matter? Diversity and Distributions 13:203-212. https://doi.org/10.1111/j.1472-4642.2006.00312.x.

Corbelli, J. M., G. A. Zurita, J. Filloy, J. P. Galvis, N. I. Vespa, and I. Bellocq. 2015. Integrating taxonomic, functional and phylogenetic beta diversities: Interactive effects with the biome and land use across taxa. PLoS ONE 10:1-17. https://doi.org/10.1371/journal.pone.0126854.

Cornwell, W. K., and D. D. Ackerly. 2009. Community assembly and shifts in plant trait distributions across an environmental gradient in coastal California. Ecological Monographs 79:109-126. https://doi.org/10.1890/071134.1.

Croci, S., A. Butet, and P. Clergeau. 2008. Does urbanization filter birds on the basis of their biological traits? The Condor 110:223-240. https://doi.org/10.1525/cond.2008.8409.

de la Peña, M. R. 2013. Nidos y reproducción de las Aves Argentinas. Ediciones Biológica. Serie Naturaleza, Conservación y Sociedad N 8 . Santa Fe, Argentina.

de la Peña, M. R. 2016. Aves argentinas: descripción, comportamiento, reproducción y distribución. Comunicaciones del Museo Provincial de Ciencias Naturales "Florentino Ameghino" (Nueva Serie) 20:1-620.

del Hoyo, J., A. Elliott, J. Sargatal, D. A. Christie, and E. de Juana. 2016. Handbook of the birds of the world alive. Lynx Editions. Barcelona, Spain.

Di Bitetti, M. S., G. Placci, and L. A. Dietz. 2003. A biodiversity vision for the Upper Paraná Atlantic Forest ecoregion: Designing a biodiversity conservation landscape and setting priorities for conservation. Fundación Vida Silvestre Argentina, World Wildlife Foundation. Washington, D.C., USA.

Díaz, S., and M. Cabido. 2001. Vive la difference: plant functional diversity matters to ecosystem processes: plant functional diversity matters to ecosystem processes. Trends in Ecology and Evolution 16:646-655. https://doi.org/ 10.1016/S0169-5347(01)02283-2.

Diniz-Filho, J. A. F., M. V. Cianciaruso, T. F. Rangel, and L. M. Bini. 2011. Eigenvector estimation of phylogenetic and functional diversity. Functional Ecology 25:735-744. https://doi.org/10.1111/j.1365-2435.2011.01836.x.

Farwig, N., N. Sajita, and K. Böhning-Gaese. 2008. Conservation value of forest plantations for bird communities in western Kenya. Forest Ecology and Management 255:3885-3892. https://doi.org/10.1016/j.foreco.2008.03.042.

Filloy, J., G. A. Zurita, and M. I. Bellocq. 2018. Bird diversity in urban ecosystems: The role of the biome and land use along urbanization gradients. Ecosystems 22:213-227. https://doi.org/10.1007/s10021-018-0264-y.

Filloy, J., G. A. Zurita, J. M. Corbelli, and M. I. Bellocq. 2010. On the similarity among bird communities: Testing the influence of distance and land use. Acta Oecologica 36:333-338. https://doi.org/10.1016/j.actao.2010.02.007.

Flynn, D. F. B., M. Gogol-Prokurat, T. Nogeire, N. Molinari, B. T. Richers, B. B. Lin, N. Simpson, M. M. Mayfield, and F. DeClerck. 2009. Loss of functional diversity under land use intensification across multiple taxa. Ecology Letters 12:22-33. https://doi.org/10.1111/j.1461-0248.2008.01255.x.

Galindo Leal, C., and I. de Gusmão Câmara. 2003. The Atlantic Forest of South America: biodiversity status, threats, and outlook. Island Press, Washington, D.C., USA.

Garaffa, P. I., J. Filloy, and M. I. Bellocq. 2009. Bird community responses along urban-rural gradients: Does the size of the urbanized area matter? Landscape and Urban Planning 90:33-41. https://doi.org/10.1016/j.landurbplan.20 08.10.004.

Gascon, C., T. E. Lovejoy, R. O. Bierregaard, J. R. Malcolm, P. C. Stouffer, H. L. Vasconcelos, W. F. Laurance, B. Zimmerman, M. Tocher, and S. Borges. 1999. Matrix habitat and species richness in tropical forest remnants. Biological Conservation 91:223-229. https://doi.org/10.1016/S0006-3207(99)00080-4.

Hausner, V. H., N. G. Yoccoz, and R. A. Ims. 2003. Selecting indicator traits for monitoring land use impacts: Birds in Northern Coastal Birch Forests. Ecological Applications 13:999-1012. https:/ / doi.org/10.1890/1051-0761(2003)13[999: 
SITFML]2.0.CO;2.

Hothorn, T., F. Bretz, and P. Westfall. 2008. Simultaneous inference in general parametric models. Biometrical Journal 50:346-363. https://doi.org/10.1002/bimj.200810425.

INBIO. 2008. Superficie Cultivada con Soja. URL: www.inbio.org.py.

Instituto Forestal Nacional. 2013. Programa de apoyo a las exportaciones paraguayas. Proyecto "Promoción de las plantaciones forestales". Informe Final. Instituto Forestal Nacional, Facultad de Ciencias Agrarias y Asociación Rural del Paraguay. San Lorenzo, Paraguay.

Jaccard, P. 1912. The distribution of the flora in the Alpine zone. New Phytologist 11:37-50. https://doi.org/10.1111/ j.1469-8137.1912.tb05611.x.

Kindt, R., and R. Coe. 2005. Tree diversity analysis: a manual and software for common statistical methods for ecological and biodiversity studies. World Agroforestry Centre. Nairobi, Kenia.

Kraft, N. J. B., P. B. Adler, O. Godoy, E. C. James, S. Fuller, and J. M. Levine. 2015. Community assembly, coexistence and the environmental filtering metaphor. Functional Ecology 29:592-599. https://doi.org/10.1111/1365-2435.12345.

Laliberté, E., and P. Legendre. 2010. A distance-based framework for measuring functional diversity from multiple traits. Ecology 91:299-305. https://doi.org/10.1890/08-2244.1.

Lawton, J. H., D. E. Bignell, B. Bolton, G. F. Bloemers, P. Eggleton, H. P. M, M. Hodda, R. D. Holt, T. B. Larsen, N. A. Mawdsley, N. E. Stork, D. S. Srivastava, and A. D. Watt. 1998. Biodiversity inventories, indicator taxa and effects of habitat modification in tropical forest. Nature 391:72-76. https://doi.org/10.1038/34166.

López-Lanús, B., P. Grilli, A. S. Di Giacomo, E. E. Coconier, and R. Banchs. 2008. Categorización de las aves de la Argentina según su estado de conservación. Informe. Aves Argentinas/Asociación Ornitológica del Plata y Secretaría de Ambiente y Desarrollo Sustentable, Buenos Aires, Argentina.

Loyn, R. H., E. G. McNabb, P. Macak, and P. Noble. 2007. Eucalypt plantations as habitat for birds on previously cleared farmland in south-eastern Australia. Biological Conservation 137:533-548. https://doi.org/10.1016/ j.biocon.2007.03.012.

Luck, G. W., A. Carter, and L. Smallbone. 2013. Changes in bird functional diversity across multiple land uses: Interpretations of functional redundancy depend on functional group identity. PLoS ONE 8:e63671. https://doi.org/ 10.1111/j.1365-2656.2012.01974.x.

Luck, G. W., S. Lavorel, S. Mcintyre, and K. Lumb. 2012. Improving the application of vertebrate trait-based frameworks to the study of ecosystem services. Journal of Animal Ecology 81:1065-1076. https://doi.org/10.1111/j.13652656.2012.01974.x.

Lundberg, J., and F. Moberg. 2003. Mobile link organisms and ecosystem functioning: Implications for ecosystem resilience and management. Ecosystems 6:87-98. https://doi.org/10.1007/s10021-002-0150-4.

MacArthur, R. H., and J. W. MacArthur. 1961. On bird species diversity. Ecology 42:594-598. https:/ / doi.org/10.2307/ 1932254.

Magurran, A. E. 2004. Measuring biological diversity. Blackwell, Oxford, UK.

Mason, N. W. H., D. Mouillot, W. G. Lee, and J. B. Wilson. 2005. Functional richness, functional evenness and functional divergence: The primary components of functional diversity. Oikos 111:112-118. https://doi.org/10.1111/j.00301299.2005.13886.x.

Mayfield, M. M., S. P. Bonser, J. W. Morgan, I. Aubin, S. McNamara, and P. A. Vesk. 2010. What does species richness tell us about functional trait diversity? Predictions and evidence for responses of species and functional trait diversity to land-use change. Global Ecology and Biogeography 19:423-431. https://doi.org/10.1111/j.1466-8238.2010.00532.x.

Millennium Ecosystem Assessment. 2005. Ecosystems and human well-being: biodiversity synthesis. World Resources Institute, Washington, D.C. USA.

Morse, N. B., P. A. Pellissier, E. N. Cianciola, R. L. Brereton, M. M. Sullivan, and K. Nicholas. 2014. Novel ecosystems in the Anthropocene: a revision of the novel ecosystem concept for pragmatic applications. Ecology and Society 19: 12. https://doi.org/10.5751/ES-06192-190212.

Mueller-Dombois, D., and H. Ellenberg. 1974. Aims and methods of vegetation ecology. John Wiley, New York, USA.

Myers, N., R. A. Mittermeier, C. G. Mittermeier, G. A. B. da Fonseca, and J. Kent. 2000. Biodiversity hotspots for conservation priorities. Nature 403:853-858. https://doi.org/10.1038/35002501.

Narosky, S., D. Yzurieta, and H. Matarasso. 2010. Aves de Argentina y Uruguay: guía de identificación. Birds of Argentina and Uruguay: a field guide. Vazquez Mazzini. Buenos Aires, Argentina.

Newbold, T., J. P. W. Scharlemann, S. H. M. Butchart, Ç. H. Pekercioðlu, L. Joppa, R. Alkemade, and D. W. Purves. 2014. Functional traits, land-use change and the structure of present and future bird communities in tropical forests. Global Ecology and Biogeography 23:1073-1084. https://doi.org/10.1111/geb.12186.

Oksanen, J., F. G. Blanchet, R. Kindt, P. Legendre, P. R. Minchin, R. B. O’Hara, G. L. Simpson, P. Solymos, M. H. H. Stevens, and H. Wagner. 2016. vegan: community ecology package. R package version 2.0-7. R Foundation for Statistical Computing, Vienna, Austria.

Oliveira-Filho, A. T., and M. A. L. Fontes. 2000. Patterns of floristic differentiation among Atlantic Forests in Southeastern Brazil and the influence of climate. Biotropica 32:793-810. https://doi.org/10.1646/0006-3606(2000)032[0793: POFDAA]2.0.CO;2. https://doi.org/10.1111/j.1744-7429.2000.tb00619.x.

Palacio, F. X., L. M. Ibañez, R. E. Maragliano, and D. Montalti. 2018. Urbanization as a driver of taxonomic, functional, and phylogenetic diversity losses in bird communities. Canadian Journal of Zoology 96:1114-1121. https://doi.org/ 
10.1139/cjz-2018-0008.

Peh, K. S. H., N. S. Sodhi, J. de Jong, C. H. Sekercioglu, C. A. M. Yap, and S. L. H. Lim. 2006. Conservation value of degraded habitats for forest birds in southern Peninsular Malaysia. Diversity and Distributions 12:572-581. https: //doi.org/10.1111/j.1366-9516.2006.00257.x.

Petchey, O. L., A. Hector, and K. J. G. Gaston. 2004. How do different measures of functional diversity perform? Ecology 85:847-857. https://doi.org/10.1890/03-0226.

Petchey, O. L., and K. J. Gaston. 2007. Dendrograms and measuring functional diversity. Oikos 116:1422-1426. https: //doi.org/10.1111/j.0030-1299.2007.15894.x.

Petit, L. J., and D. R. Petit. 2003. Evaluating the importance of human-modified lands for neotropical bird conservation. Conservation Biology 17:687-694. https://doi.org/10.1046/j.1523-1739.2003.00124.x.

Pinheiro, J., D. Bates, S. DebRoy, and D. Sarkar. 2014. R Core Team. nlme: Linear and Nonlinear mixed effects models. R package version 3.1-117. URL: CRAN.R-project.org/package=nlme

R Core Team. 2018. R: A Language and Environment for Statistical Computing. R Foundation for Statistical Computing. URL: www.r-project.org.

Rakotoarisoa, J.-E., and A. P. Capparella. 2013. Forest-bird species assemblages in the lowland rainforest of Madagascar: The effects of forest fragmentation revisited. The Open Conservation Biology Journal 7:27-41. https:/ / doi.org/10.2174/ 1874839201307010027.

Ralph, C. J., G. R. Geupel, P. Pyle, T. E. Martin, D. F. DeSante, B. Milá, C. John, R. Geoffrey, E. Thomas, and F. David. 1996. Manual de métodos de campo para el monitoreo de aves terrestres. Pacific Southwest Research Station, Forest Service, U.S. Department of Agriculture, Albany, CA. https://doi.org/10.2737/PSW-GTR-159.

Ribeiro, M. C., J. P. Metzger, A. C. Martensen, F. J. Ponzoni, and M. M. Hirota. 2009. The Brazilian Atlantic Forest: How much is left, and how is the remaining forest distributed? Implications for conservation. Biological Conservation 142 : 1141-1153. https://doi.org/10.1016/j.biocon.2009.02.021.

Sayer, C. A., J. M. Bullock, and P. A. Martin. 2017. Dynamics of avian species and functional diversity in secondary tropical forests. Biological Conservation 211:1-9. https://doi.org/10.1016/j.biocon.2017.05.004.

Schleuter, D., M. Daufresne, F. Massol, and C. Argillier. 2010. A user's guide to functional diversity indices. Ecological Monographs 80:469-484. https://doi.org/10.1890/08-2225.1.

Sekercioglu, C. H. 2006. Increasing awareness of avian ecological function. Trends in Ecology and Evolution 21:464-471. https://doi.org/10.1016/j.tree.2006.05.007.

Sekercioglu, C. H. 2012. Bird functional diversity and ecosystem services in tropical forests, agroforests and agricultural areas. Journal of Ornithology 153:153-161. https://doi.org/10.1007/s10336-012-0869-4.

Sekercioglu, C. H., G. C. Daily, and P. R. Ehrlich. 2004. Ecosystem consequences of bird declines. Proceedings of the National Academy of Sciences 101:18042-18047. https://doi.org/10.1073/pnas.0408049101.

Sekercioglu, C. H., S. R. Loarie, F. Oviedo Brenes, P. R. Ehrlich, and G. C. Daily. 2007. Persistence of forest birds in the Costa Rican agricultural countryside. Conservation Biology 21:482-494. https://doi.org/10.1111/j.15231739.2007.00655.x.

Seress, G., and A. Liker. 2015. Habitat urbanization and its effects on birds. Acta Zoologica Academiae Scientiarum Hungaricae 61:373-408. https:/ / doi.org/10.17109/AZH.61.4.373.2015.

Stein, A., K. Gerstner, and H. Kreft. 2014. Environmental heterogeneity as a universal driver of species richness across taxa, biomes and spatial scales. Ecology Letters 17:866-880. https://doi.org/10.1111/ele.12277.

Swenson, N. G. 2011. Phylogenetic beta diversity metrics, trait evolution and inferring the functional beta diversity of communities. PLoS ONE 6:e21264. https://doi.org/10.1371/journal.pone.0021264.

Swenson, N. G., P. Anglada-Cordero, and J. A. Barone. 2011. Deterministic tropical tree community turnover: evidence from patterns of functional beta diversity along an elevational gradient. Proceedings of the Royal Society B: Biological Sciences 278:877-884. https:/ / doi.org/10.1098/rspb.2010.1369.

Tabarelli, M., A. V. Aguiar, M. C. Ribeiro, and J. P. Metzger. 2010. Prospects for biodiversity conservation in the Atlantic Forest: Lessons from aging human-modified landscapes. Biological Conservation 143:2328-2340. https: //doi.org/10.1016/j.biocon.2010.02.005.

Temperton, V. M. 2004. Assembly rules and restoration ecology: Bridging the gap between theory and practice. Island Press. Washington, D.C., USA.

Tilman, D. 2001. Functional diversity. In Encyclopedia of biodiversity. Academic Press, San Diego, California, USA. https://doi.org/10.1016/B978-0-12-384719-5.00061-7.

Tinoco, B. A., V. E. Santillán, and C. H. Graham. 2018. Land use change has stronger effects on functional diversity than taxonomic diversity in tropical Andean hummingbirds. Ecology and Evolution 8:3478-3490. https://doi.org/ 10.1002/ece3.3813.

Tscharntke, T., C. H. Sekercioglu, T. V. Dietsch, N. S. Sodhi, P. Hoehn, and J. M. Tylianakis. 2008. Landscape constraints on functional diversity of birds and insects in tropical agroecosystems. Ecology 89:944-951. https://doi.org/10.1890/ 07-0455.1.

Vaccaro, A., J. Filloy, and M. Bellocq. 2019. What land use better preserves the functional and taxonomic diversity of birds in a grassland biome? Avian Conservation and Ecology 14:1. https://doi.org/10.5751/ACE-01293-140101.

Van Bael, S. A., J. D. Brawn, and S. K. Robinson. 2003. Birds defend trees from herbivores in a Neotropical forest canopy. Proceedings of the National Academy of Sciences of the United States of America 100:8304-7. https:// doi.org/10.1073/pnas.1431621100. 
Vandewalle, M., F. de Bello, M. P. Berg, T. Bolger, S. Dolédec, F. Dubs, C. K. Feld, R. Harrington, P. A. Harrison, S. Lavorel, P. M. da Silva, M. Moretti, J. Niemelä, P. Santos, T. Sattler, J. P. Sousa, M. T. Sykes, A. J. Vanbergen, and B. A. Woodcock. 2010. Functional traits as indicators of biodiversity response to land use changes across ecosystems and organisms. Biodiversity and Conservation 19:2921-2947. https://doi.org/10.1007/s10531-010-9798-9.

Villéger, S., N. W. H. Mason, and D. Mouillot. 2008. New multidimensional functional diversity indices for a multifaceted framework in functional ecology. Ecology 89:2290-2301. https://doi.org/10.1890/07-1206.1.

Violle, C., M. L. Navas, D. Vile, E. Kazakou, C. Fortunel, I. Hummel, and E. Garnier. 2007. Let the concept of trait be functional! Oikos 116:882-892. https://doi.org/10.1111/j.2007.0030-1299.15559.x.

Waltert, M., A. Mardiastuti, and M. Mühlenberg. 2004. Effects of land use on bird species richness in Sulawesi, Indonesia. Conservation Biology 18:1339-1346. https:/ /doi.org/10.1111/j.1523-1739.2004.00127.x.

Wotton, D. M., and D. Kelly. 2011. Frugivore loss limits recruitment of large-seeded trees. Proceedings of the Royal Society B: Biological Sciences 278:3345-3354. https:/ / doi.org/10.1098/rspb.2011.0185.

Xeno-canto-Foundation. 2018. Xeno-canto website. URL: www.xeno-canto.org.

Zipperer, W. C., S. S. Sisinni, R. R. Pouyat, and T. T. Foresman. 1997. Urban tree cover: an ecological perspective. Urban Ecosystems 1:229-246. https:/ /doi.org/10.1023/A:1018587830636.

Zurita, G. A., and M. I. Bellocq. 2010. Spatial patterns of bird community similarity: Bird responses to landscape composition and configuration in the Atlantic forest. Landscape Ecology 25:147-158. https:/ /doi.org/10.1007/s10980009-9410-4.

Zurita, G. A., and M. I. Bellocq. 2012. Bird assemblages in anthropogenic habitats: identifying a suitability gradient for native species in the Atlantic Forest. Biotropica 44:412-419. https://doi.org/10.1111/j.1744-7429.2011.00821.x. 